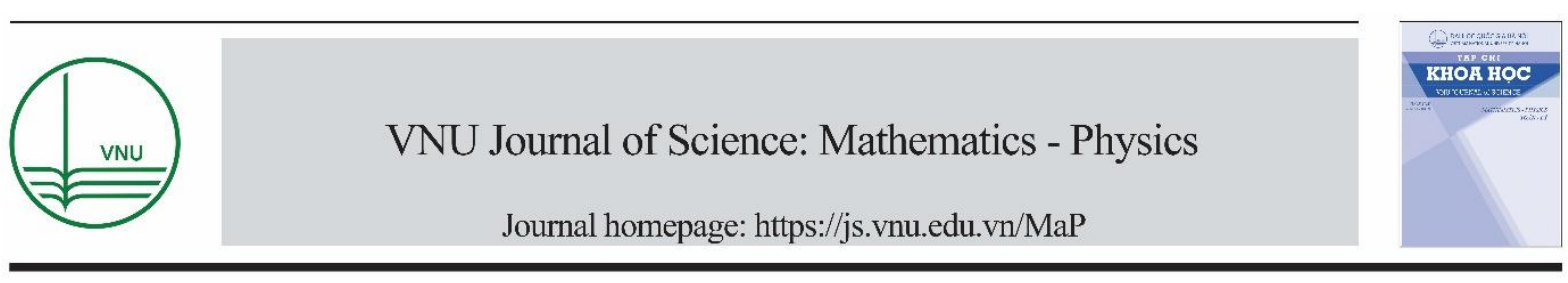

Original Article

\title{
The Enhancement of Visible Absorption of Cu Doped ZnO Nanorods on Patterned Substrates
}

\author{
My Hoa Tong, ${ }^{1}$ Thi Hoa Lai, ${ }^{2.3}$ Nhat Minh Nguyen, ${ }^{1}$ Thi Kieu Hanh Ta, ${ }^{1,2,3}$ \\ Thanh Tuan Anh Pham, ${ }^{1,3}$ Tran My Hoa Huynh, ${ }^{1,3}$ Cong Khanh Tran ${ }^{1,3}$ \\ Bach Thang Phan ${ }^{2,3}$, Vinh Quang Dang ${ }^{1,2,3^{*}}$ \\ ${ }^{1}$ University of Science, 227 Nguyen Van Cu street, Ward 4, District 5, Ho Chi Minh city, Viet Nam \\ ${ }^{2}$ Center for Innovative Materials and Architectures (INOMAR), Quarter 6, \\ Linh Trung, Thu Duc District, Ho Chi Minh City \\ ${ }^{2}$ Vietnam National University, Ho Chi Minh City (VNU-HCM), Linh Trung, \\ Thu Duc, Ho Chi Minh city, Viet Nam
}

Received 26 June 2020

Revised 21 July 2020; Accepted 15 August 2020

\begin{abstract}
We report the antireflection and light absorption in visible region by new stretchable substrates with patterned structure. Mogul substrates with 3-Dimentional structures were fabricated by using polydimethyl - siloxane that imitate the nanostructures surface. Then, Copper doped $\mathrm{ZnO}$ NRs on mogul-patterned surface by hydrothermal method at low temperature. The optical properties, morphology and structures of $\mathrm{ZnO}: \mathrm{Cu} \mathrm{NRs}$ were investigated through out of measurement the scanning electron microscopy, X-Ray diffraction and ultraviolet-visible spectroscopy, respectively. The results show the $\mathrm{Cu}$ doped $\mathrm{ZnO}$ NRs were uniformly and dense grown on mogul substrates, well oriented in the (002) plane. Additionally, the light absorption can be significantly enhanced to more $10 \%$ in a wide spectral range $(400-800 \mathrm{~nm})$ due to the reduce reflection. Growing ZnO NRs doping on new stretchable substrates with a mogul-patterned surface were successfully fabricated and applicable in the flexible and stretchable optoelectronic devices.
\end{abstract}

Keywords: doping, patterned surface, mogul, $\mathrm{ZnO}$ NRs, visible absorption.

\footnotetext{
${ }^{*}$ Corresponding author.

Email address: vinhquangntmk@gmail.com
}

https//doi.org/ 10.25073/2588-1124/vnumap.4569 


\section{Introduction}

Optoelectronic devices (ODs) have become an important part of our life. Recently, the development of fabrication and application of ODs are being widely researched. Wherever light is used to transmit information, tiny semiconductor devices are needed to transfer electrical current into optical signals and vice versa. Examples include light emitting diodes in radios and other appliances, photodetector in elevator doors and digital cameras, and laser diodes that transmit phone calls through glass fibers[1].

ODs typically use direct band gap materials such as II- IV semiconductor and light often includes invisible forms of radiation such as gamma rays, X-rays, ultraviolet and infrared, in addition to visible light[1]. ODs are electrical-to-optical or optical-to-electrical transducers or instrument that use such device their operation[1]. The Zinc oxide nanorods ( $\mathrm{ZnO} \mathrm{NRs)}$ are one of the most potential semiconductor materials that used in ODs due to their optical and electrical properties. ZnO NRs are very high absorption in the ultraviolet visible (UV) region, and large surface-to-volume to radio and variety of morphologies and availability of simple and low cost processing[2]. Currently, many researchers focused studying in this material and applying in ODs such as optical sensor[3], lightemitting devices, flexible electronics and solar cells[4]. However, ZnO NRs has wide band gap (band gap energy $=3.37 \mathrm{eV}$ ) and high exciton binding energy of $60 \mathrm{meV}$ at room temperature so absorption incident light at visible region is extremely low[5].

Nowadays, many researches effort to develop ODs such as experimental studies of transition metal doped $\mathrm{ZnO}$ have been reported, including studies of doped nanostructures can increase the effective absorption path of the incident light. Commonly used dopants are $\mathrm{Co}, \mathrm{Ni}[6], \mathrm{Cu}, \mathrm{Mn}[7]$, and $\mathrm{Cr}[8]$. For examples, $\mathrm{Cu}$ doped $\mathrm{ZnO}$ photocatalyst thin film were prepared by the sol-gel dip-coating method. This research $\mathrm{Cu}$ doped $\mathrm{ZnO}$ was fabricated on glass substrates and the result that showed all $\mathrm{ZnO}$ which is prepared very high transmittance above $89 \%$ in the visible region $(400-800 \mathrm{~nm})$. The observed increase in the optical band gap energy as the initial $\mathrm{Cu}$ concentration increased was caused by a decrease of the grain size. The decrease of the grain size produced by increasing the dopant concentrations was explained by the pinning effect. On the other hand, $\mathrm{Ag}$ was decorated on $\mathrm{ZnO}$ NRs substrates to increase absorption in visible region by effect plasmon surface[9,10]. However, the performance and efficiency in this device low and unstable was caused by a fast recombination reaction, response for detector and recovery time still slowly[11]. Additionally, the common problem with doped nanostructures that change of morphology so many research groups have developed various materials and device technologies on stretchable substrates to improve device performance, simple process, low cost and enhance absorption of $\mathrm{ZnO}$ NRs. For example, the development of highly sophisticated flexible mobile phones and wearable computing devices or the antireflection and light absorption enhancement by forming sub-wavelength or nano-patterned Si structures via nano-sphere lithography technique[12]. In adition, antireflection techniques, such as modified nano patterned structures and the antireflection coatings, have been used to reduce the reflection loss in order to improve performance[13].

In this work, patterned substrates are replicated by using polydimethyl-siloxane (PDMS) pour on nanostructure surface and the $\mathrm{ZnO}$ nanorods (NRs) are grown on these various 3-Dimentional (3-D) structures using hydrothermal method at low temperature to study their optical properties. Various methods have been developed to produce one-dimensional $\mathrm{ZnO}$ nano materials, including sol-gel methods, polymer-assisted growth, template-induced growth, solution-liquid-solid growth in an organic solvent, metal-organic chemical vapor deposition (MOCVD) and hydrothermal synthesis. However, the hydrothermal method has many advantages over other method such as relatively simple process, low synthesis temperature, controllable size and low cost[14]. The morphology, structure and optical properties of $\mathrm{ZnO}$ NRs grown patterned substrate are investigate by scanning electron microscopy (SEM), X-ray diffraction (XRD) and Ultraviolet-Visible spectroscopy (UV-Vis), respectively. The 
results show the high density $\mathrm{ZnO}$ NRs, well oriented in the (002) plane and increased absorption in visible region due to the reduction of light reflection on the patterned substrates. The reflection can be reduced to less than $20 \%$ and the absorption can be enhanced to more $10 \%$. Growing ZnO NRs on the 3-D structure at low temperature enable the potential applications such as flexible and stretchable optoelectronic devices using visible light.

\section{Materials - Methods}

\subsection{Chemical Materials}

Zinc oxide nanoparticles $(\mathrm{ZnO} N P s)$ and Copper nitrate trihydrate $\left(\mathrm{Cu}\left(\mathrm{NO}_{3}\right)_{2} .3 \mathrm{H}_{2} \mathrm{O}\right)$ were bought from Sigma Aldrich. Poly(dimethysiloxane) (PDMS) was ordered from Dow Chemical. Zinc nitrate hexahydrate $\left(\left(\mathrm{Zn}\left(\mathrm{NO}_{3}\right)_{2}\right) \cdot 6 \mathrm{H}_{2} \mathrm{O}\right)$ and Hexamethylene tetramine $(\mathrm{HTM})$ were bought from Xilong Scienctific

\subsection{Synthesized process}

\subsubsection{The cleaner Patterned Substrates}

The glass substrates were cleaned by successive ultrasonic cleaning in acetone, ethanol and DI water, for each $20 \mathrm{~min}$ to remove grease, soluble organic compounds and other contaminants that could affect the quality of the $\mathrm{ZnO}$ NRs from its surface then dried.

\subsubsection{Replication of the Mogul-Patterned PDMS Substrate}

The base and curing agent is mixed with weight or volume ratio of 10:1. The elastomer PDMS was poured on the patterned substrate and kept in a vacuum chamber to remove any air bubbles. Next, the sample was heated at $80^{\circ}$ for $1 \mathrm{~h} 30$ min that the air bubbles do not return and PDMS film can promptly dry. After that, PDMS was detached from mogul patterned on the glass.

\subsubsection{Preparation of $\mathrm{ZnO}$ Seed Layers}

The ZnO NPs were first dissolved in the solvent ethanol with stirring for completing dissolution. $\mathrm{ZnO}$ NPs were diluted to $2 \%$ in ethanol. Next, APTES was mixed with the isopropanol. Then, this solution was dropped on the mogul-patterned PDMS substrate that surface of patterned substrates become the hydrophilic. The solution ZnO NPs and ethanol was spin coated at a rate of $3000 \mathrm{rpm}$ for 30 s using a spin coater. The as-deposited thin film was heated in an oven at $90^{\circ} \mathrm{C}$ for $10 \mathrm{~min}$ to remove the solvent. After repeating the spin coating and drying procedures for two times to yield the required thickness, the resulting thin film was annealed at $90^{\circ} \mathrm{C}$ in $30 \mathrm{~min}$ to obtain the $\mathrm{ZnO}$ seed layer.

\subsubsection{Preparation of Cu-doped $\mathrm{ZnO}$ Nanorods}

After the formation of seed layer, $\mathrm{Cu}$ doped $\mathrm{ZnO}$ NRs were prepared by dissolving $\mathrm{Cu}\left(\mathrm{NO}_{3}\right)_{2} .3 \mathrm{H}_{2} \mathrm{O}$ in aqueous of $\left(\mathrm{Zn}\left(\mathrm{NO}_{3}\right)_{2}\right) \cdot 6 \mathrm{H}_{2} \mathrm{O}$ and $\mathrm{HMT}$ at room temperature. After the preparation of $\mathrm{Cu}$ optimize concentration is $3 \%$ by volume, the substrates with a seed layer were put upside down in a glass beaker filled with the above solution, sealed, heated at $90^{\circ} \mathrm{C}$ for $4 \mathrm{~h}$, then cleaned with distilled water and dried at $90^{\circ} \mathrm{C}$ for $30 \mathrm{~min}$.

\subsubsection{Materials characteristics}

Morphological characterization and elemental analysis of the NRs were performed in FE SEM S4800 Hitachi. The crystal structures of ZnO NRs are examined in Bruker D8 Advance Powder X-Ray Diffractometer with $\mathrm{Cu} k \alpha$ radiation of $1.541 \AA$ A. Optical properties of $\mathrm{ZnO}$ NRs grown patterned 
substrate are investigated by Ultraviolet-Visible spectroscopy (UV-Vis) by UV-Vis Schimadzu UV1800 .

\section{Results and Discussions}

\subsection{Crystal Structure Cu Doped ZnO NRs}
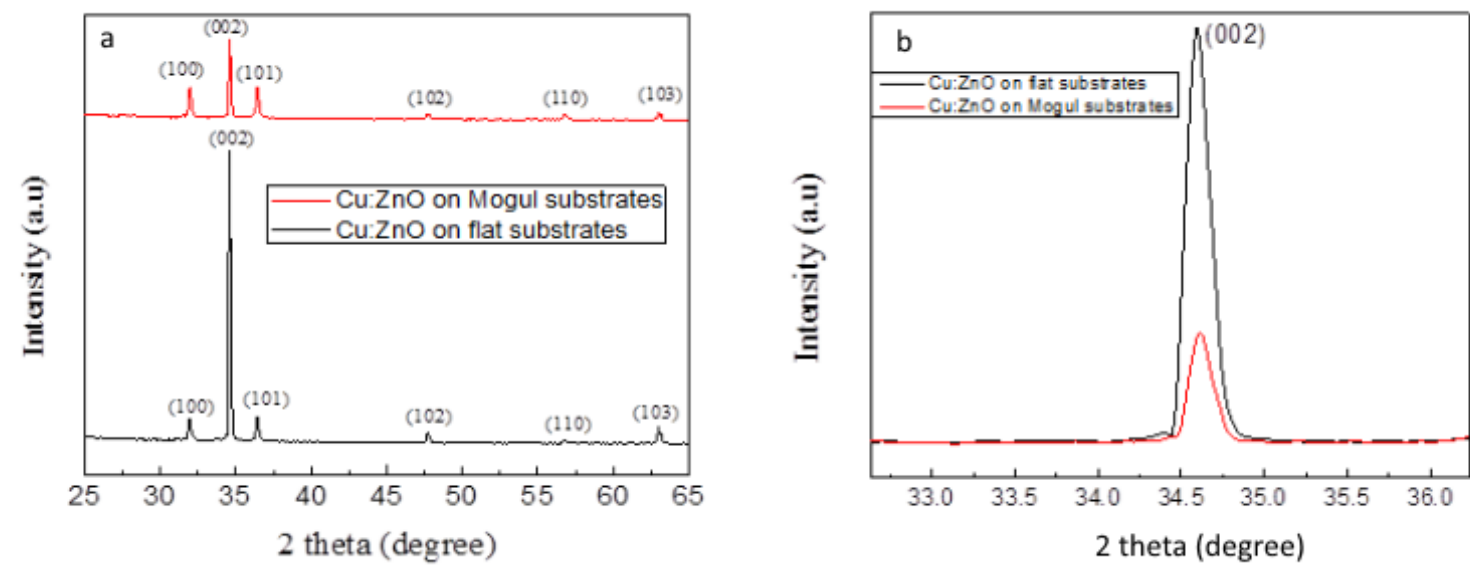

Figure 1. (a) The XRD pattern of $\mathrm{Cu}$ doped $\mathrm{ZnO}$ NRs grown on the flat surface and patterned substrate.

(b) Comparison of the (002) peaks.

Structural characterization of $\mathrm{Cu}$ doped $\mathrm{ZnO}$ NRs was done by XRD measurement. The crystallite characteristics of these NRs demonstrated the hexagonal Wurtzite structures. ZnO NRs characteristics have six peak that can be seen as $31.76^{\circ}, 36.21^{\circ}, 47.59^{\circ}, 56.76^{\circ}$, which correspond to (100), (101), (102), (110) and (103). The highest intensity of the XRD peak is (002) at $34.4^{\circ}$, which further confirmed the highly crystalline nature of the sample and verifies the NRs growth preferential orientation on the caxis. It is found that the peak position is slightly shifted towards higher $2 \theta$. The result in Figure 1 shows $\mathrm{Cu}$ phases still have not appeared within sensitivity of the XRD which implies that $\mathrm{Cu}$ doped $\mathrm{ZnO}$ NRs do not still affect to Wurtzite crystallite characteristics structure.

We can see that the intensity of (002) peak on flat substrates higher than that on mogul substrates because $\mathrm{ZnO}$ NRs on flat substrates have crystallize better than patterned substrates. Because patterned substrates have wavelength nano-structures so $\mathrm{ZnO}$ grain in grow process it can be agglomerate, low crystallization.

The average nano crystalline size was calculated using Debye-Scherrer's formula through the value of X-ray diffraction spectra[11]:

$$
D=\frac{k \lambda}{\beta_{h k l} \cos \theta}
$$

Where $\mathrm{D}=$ crystallite size $(\mathrm{nm}), \mathrm{k}=$ shape factor $(0.9)$, and $\beta_{h k l}$ is the full width at half maximum (FWHM-rad), $\theta=$ diffraction angle and $\lambda$ is the wavelength of monochromatic $\mathrm{Cu}-\mathrm{K} \alpha$ irradiation using in the XRD measurement system $(\lambda=1.541 \AA)[11]$.

The diameter calculated by Debye-Scherrer's formula is approximately $38 \mathrm{~nm}$ with $\mathrm{Cu}$ doped $\mathrm{ZnO}$ NRs on flat substrates and that on the Mogul substrates is around $39 \mathrm{~nm}$. The reason for difference size 
between various substrates is explained by agglomerate. Pattern substrates have valleys and bumps in a periodic fashion so structures patterned substrates can be seen like the trap. During growth process, $\mathrm{ZnO}$ NRs have agglomerate together tendency in hole. This is lead to crystallize grain will priority grow $(002)$ planes so the grains size larger than flat substrates that the crystalline of ZnO NRs not better than flat substrates.

\subsection{Morphology of Cu Doped ZnO NRs}

Figure 2a shows field-emission scanning electron microscopy (SEM) images of the mogul pattern on the UV- curable polyurethane acrylate (PUA) master mold and the mogul-patterned PDMS replicated by soft lithography from the PUA master mold, respectively. We found the surface topography of the mogul-patterned PDMS replicated from a PUA master mold exhibited the same results as those made directly hat. The stretchable substrate with a mogul patterned surface which can be not only multidirectionally stretchable and versatile for various thin film materials, but also improve the reliability of structures layered on it due to the efficient absorption of applied stress and good adhesion layers on the substrates surface. Additionally, the mogul structure has valleys and bumps in a periodic fashion. Thanks to this structure of mogul substrates, the layers can reduce their stress by straightening out the mogul-pattern stretchable substrates. Figure $2 b, 2 c$ presents the top view and cross-section SEM of $\mathrm{Cu}$ doped $\mathrm{ZnO}$ NRs grown on mogul structure. The depth and breadth of the hole correspond to 50 $\mu \mathrm{m}$ and $100 \mu \mathrm{m}$. The high density and uniform of nanorods are displayed in Figure $2 \mathrm{~d}$ that demonstrated the successful growth of $\mathrm{Cu}$ doped $\mathrm{ZnO}$ NRs on patterned surfaces.
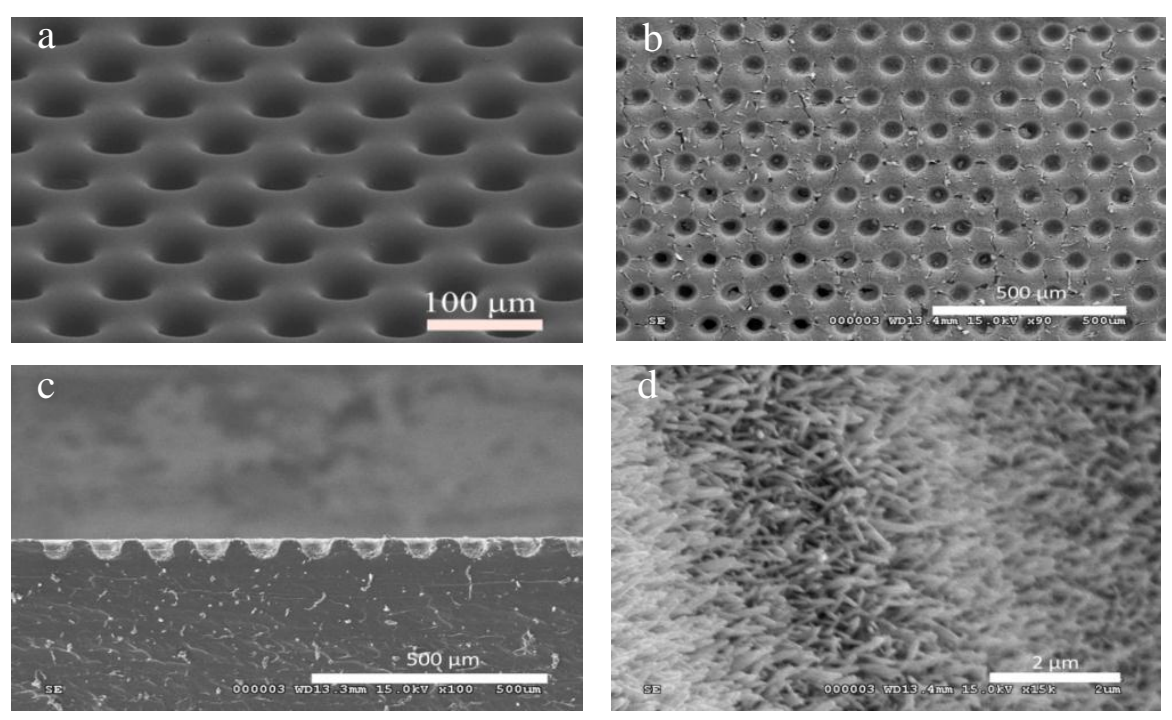

Figure 2. (a)Top view SEM image of stretchable substrates with a mogul pattern, the morphology of $\mathrm{Cu}$ doped $\mathrm{ZnO}$ NRs array on Mogul substrate (b) top view, (c) cross-section. (d) the large scale of Cu doped $\mathrm{ZnO}$ NRs on patterned surface.

\section{2. $U V$-Vis Spectroscopy}

The antireflection effect of the nano-patterned substrates was characterized by the calibrated process over a broad wavelength range $(400-800 \mathrm{~nm})$. Figure 3 a shows the reflection spectra of $\mathrm{ZnO}$ NRs on flat substrates exhibits higher than reflection on mogul substrates in the visible light region (400- 
800nm). The absorption spectra of mogul and flat substrates are can be calculated based on the reflection and transmission measurements. As given in Figure 3b, it is found that the absorption of ZnO NRs on Mogul substrates is significantly enhanced in a wide spectral range (400-800nm). Our results suggest that the patterning substrates formed by the present approach exhibit the good antireflection and absorption enhancement characterization because it provides grading refraction index at the air/PDMS interface. Meanwhile, the optical absorption can be enhanced by using sub-wavelength nano-structures. Since the feature size of surface pattern is smaller than wavelength, the incident electreomagnetic wave can be couple with the whole surface sub-wavelenght structures which can trap the light to enhance the light harvesting in a wide spectral range. Althought the aspect ratio is not so high, the good antireflection and absorption enhancement propreties reflect their possible application in the future solar cells without introducing a high level of surface defects and low carrier collection eficiency as in the high aspect-ratio structures.
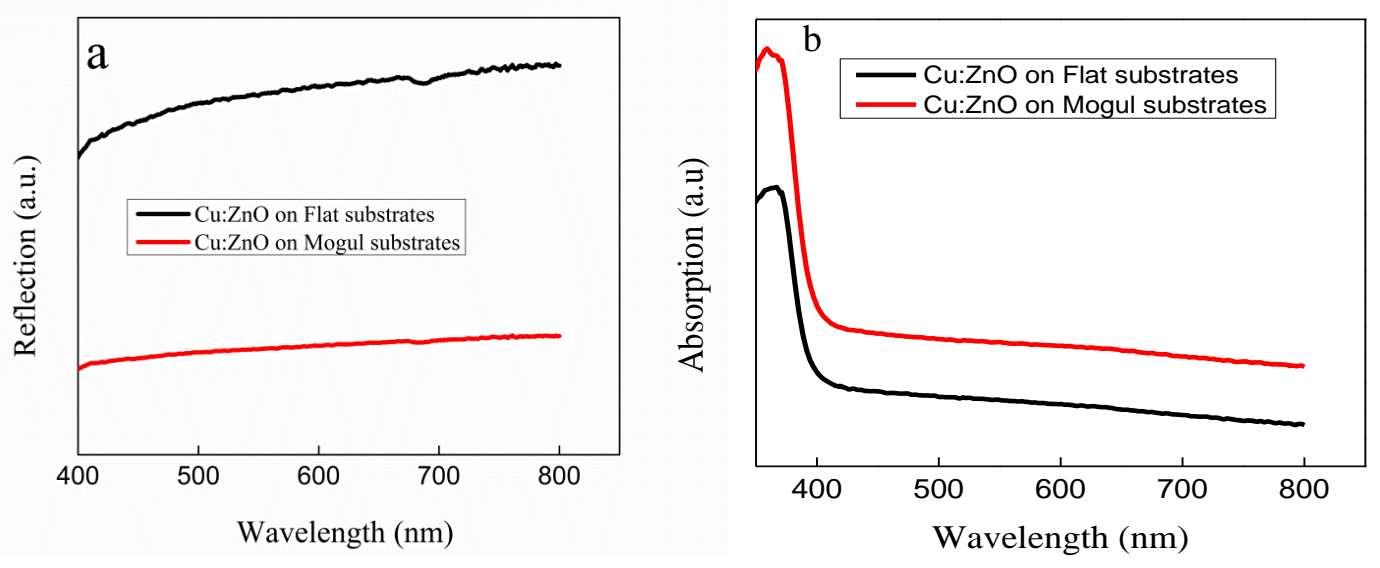

Figure 3. UV-Vis spectra of 3\% Cu doped ZnO NRs on Mogul substrates and flat substrates: a) Reflection spectra, b) Absorption spectra.

\subsection{Mechanism}

Light may be absorbed, transmitted or reflected. When the substrates are illuminated by a signal radiation, some of the light will be reflected, some is absorbed within the material and some is transmitted through it. The Figure 4 shows that light coming to flat substrates is immediately reflected contrary to mogul substrates. The different in light reflection and absorption at flat and patterned substrates can be explained by light trapping which related to wavelength nano-structures on the front of mogul substrates surface. The mogul substrate was designed bumps, valleys and opaque so forces light to bounce more than once on the substrates and thus giving multiple chances to light rays enter the substrates. This happened due to these wavelength nano substrates controls the light's travelling path by changing the angle surface in which light travels, thus giving a secondary scattering and this help keeping absorption losses in the scatters to a minimum before light way back to the air[15]. For glass substrate is thin, glossy surface and transparent and the light only transmit transparent medium. Additionally, when the reflection happened at the interface between the air and surface, the light will be bounce and way back to the air. All factors make absorption light on patterned substrates higher than glass substrates. 

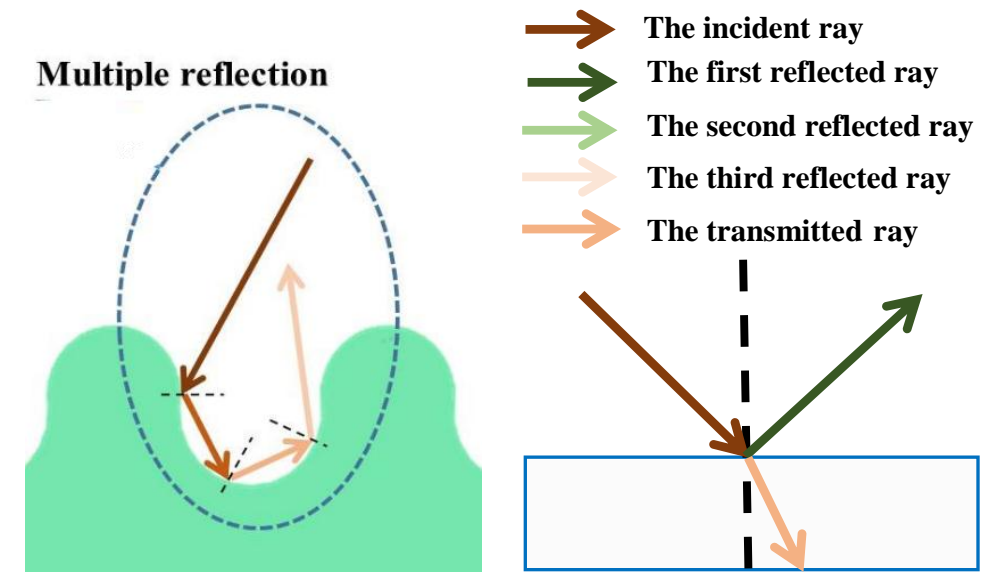

Figure 4. Simulate reflection light on patterned substrates and flat surfaces.

\section{Conclusion}

In summary, $\mathrm{ZnO}$ seeds were uniform formed on PDMS substrates by hydrothermal method, the high density $\mathrm{Cu}$ doped $\mathrm{ZnO} \mathrm{NRs}$ well oriented in the (002) plane. Our results indicated that the $\mathrm{ZnO}$ NRs doping arrays coated on patterned substrates were reduced light reflection than PDMS on flat substrates, thus enhanced absorption in visible region. The mean reflection can be reduced to less than $20 \%$ and the absorption can increase by than more $10 \%$. Growing ZnO NRs doping on the 3-D structure at low temperature enable the potential applications such as flexible and stretchable optoelectronic devices using visible light.

\section{Acknowledgments}

This research is funded by the Vietnam National Foundation for Science and Technology Development (NAFOSTED) under grant number 103.03-2018.59.

\section{References}

[1] J. Piprek, Semiconductor Optoelectronic Devices: Introduction to Physics and Simulation, Semicond. Optoelectron. Devices Introd. Phys. Simul. (2003) 1-279. https://doi.org/10.1016/C2009-0-22633-X.

[2] B. Kumar, K.Y. Lee, H. K. Park, S.J. Chae. Y. H. Lee, S. W. Kim, Controlled Growth of Semiconducting Nanowire, Nanowall, and Hybrid Nanostructures on Graphene for Piezoelectric Nanogenerators, ACS Nano, 5 (2011), 4197 4204. https://doi.org/10.1021/nn200942s.

[3] S. Dhara, P. K. Giri, Improved fast photoresponse from $\mathrm{Al}$ doped $\mathrm{ZnO}$ nanowires network decorated with $\mathrm{Au}$ nanoparticles Chem. Phys. Lett. 541 (2012) 39-43. https://doi.org/ 10.1016/j.cplett.2012.05.026.

[4] H. M. Kim, J. H. Park, S. K. Lee, Fiber optic sensor based on $\mathrm{ZnO}$ nanowires decorated by Au nanoparticles for improved plasmonic biosensor Sci. Rep. 9 (2019) 15605. https://doi.org/ 10.1038/s41598-019-52056-1.

[5] N. Liu, G. Fang, W. Zeng, H. Zhou, F. Cheng, Q, Zheng, L. Yuan, X. Zou, X, Zhao, Direct Growth of Lateral ZnO Nanorod UV Photodetectors with Schottky Contact by a Single-Step Hydrothermal Reaction, ACS Appl. Mater. Interfaces, 2 (2010) 1973-9. https://doi.org/ 10.1021/am100277q. 
[6] P. Pascariu, L. V. Tudose, M. Suchea, E. Koudoumas, N. Fifere, A. Airinei, Preparation and characterization of Ni, Co doped $\mathrm{ZnO}$ nanoparticles for photocatalytic applications, Appl. Surf. Sci. 448 (2018) 481-8. https://doi.org/10.1016/j.apsusc.2018.04.124.

[7] S. A. Ahmed, Structural, optical, and magnetic properties of Mn-doped ZnO samples Results Phys. 7 (2017) 60410. https://doi.org/10.1016/j.rinp.2017.01.018.

[8] P.E. Amami, J. Das, D.K. Mishra, V. V. Srinivasu, D.R. Sahu, B. K. Roul, Cr doped ZnO: Investigation of magnetic behaviour through SQUID and ESR studies Phys. B Condens. Matter 572 (2019) 60-5. https://doi.org/10.1016/j.physb.2019.07.056.

[9] Y. Chen, W. H. Tse, L. Chen, Z. Lin, Ag nanoparticles-decorated ZnO nanorod array on a mechanical flexible substrate with enhanced optical and antimicrobial properties Nanoscale Res. Lett. 10 (2015) 106. https://doi.org/10.1186/s11671-014-0712-3.

[10] C. Suo, C. Gao, X. Wu. Y. Zuo, Ag-decorated ZnO nanorods prepared by photochemical deposition and their high selectivity to ethanol using conducting oxide electrodes RSC Adv. 5 (2015) 92107-13. https://doi.org/ 10.1039/C5RA20055E

[11] M. Chakraborty, A. Ghosh, R. Thangavel, Experimental and theoretical investigations of structural and optical properties of copper doped $\mathrm{ZnO}$ nanorods J. Sol-Gel Sci. Technol. 3 (2015) 756-64. https://doi.org/10.1007/s10971-015-3660-1.

[12] P. Gutruf, S. Walia, A.M. N. Ali, S. Sriram, M. Bhaskaran, Strain response of stretchable micro-electrodes: Controlling sensitivity with serpentine designs and encapsulation Appl. Phys. Lett. 104 (2014) 021908. https://doi.org/10.1063/1.4862264.

[13] Y. Liu, S.H. Sun, J. Xu, L. Zhao, H. C. Sun, J. Li, W. Mu, L, Xu, K. L. Chen, Broadband antireflection and absorption enhancement by forming nano-patterned Si structures for solar cells Opt. Express 19 (2011) A1051-6. https://doi.org/10.1364/OE.19.0A1051.

[14] T. Q. Trung, V. Q. Dang, H. B. Lee, D. I. Kim, S. Moon, N. E. Lee, H. Lee, An Omnidirectionally Stretchable Photodetector Based on Organic-Inorganic Heterojunctions, ACS Appl. Mater. Interfaces 9 (2017) 35958-67. https://doi.org/10.1021/acsami.7b09411

[15] H. N. Pham, M. H. Tong, H. Q. Huynh, H. D Phan, C. K. Tran, B. T. Phan, V. Q. Dang, The Enhancement of Visible Photodetector Performance based on Mn doped ZnO Nanorods by Substrate Architecting Sens. Actuators Phys. 311 (2020) 112085. https://doi.org/10.1016/j.sna.2020.112085. 